\title{
ENTALPÍA DE DISOLUCIÓN DE SULFACETAMIDA SÓDICA EN AGUA: COMPARACIÓN ENTRE LA CALORIMETRÍA ISOPERIBÓLICA DE SOLUCIÓN Y EL MÉTODO DE VAN'T HOFF
}

\author{
Daniel R. Torres \\ Facultad de Ciencias Básicas e Ingeniería, Universidad Nacional Abierta y a Distancia, Bogotá D.C., Colombia \\ Alejandro Sosnik \\ Departamento de Tecnología Farmacéutica, Facultad de Farmacia y Bioquímica, Universidad de Buenos Aires, Consejo Nacional \\ de Investigaciones Científicas y Técnicas, Buenos Aires, Argentina \\ Diego Chiappetta \\ Departamento de Tecnología Farmacéutica, Facultad de Farmacia y Bioquímica, Universidad de Buenos Aires, Buenos Aires, \\ Argentina \\ Edgar F. Vargas \\ Departamento de Química, Facultad de Ciencias, Universidad de los Andes, Bogotá D.C., Colombia \\ Fleming Martínez* \\ Departamento de Farmacia, Facultad de Ciencias, Universidad Nacional de Colombia, A.A. 14490, Bogotá D.C., Colombia
}

Recebido em 3/10/07; aceito em 14/3/08; publicado na web em 1/9/08

\begin{abstract}
DISSOLUTION ENTHALPY OF SODIUM SULFACETAMIDE IN WATER: COMPARISON BETWEEN SOLUTION ISOPERIBOLIC CALORIMETRY AND THE VAN'T HOFF METHOD. The dissolution enthalpy $\left(\Delta H_{\text {soln }}^{0}\right.$ ) of sodium sulfacetamide in water was determined by means of isoperibolic solution calorimetry. It was found that $\Delta H^{0}{ }_{\text {soln }}$ diminishes as the drug concentration increases. Otherwise, the calorimetric values obtained as a function of the drug concentration were significantly different than those predicted by the van't Hoff method. It was demonstrated that the later is not a fully reliable method for the determination of $\Delta H^{0}{ }_{\text {soln }}$ values in the specific case of highly soluble sodium salts. The observed phenomenon could be explained by the presence of strong solute-solute interactions at high salt concentrations, in addition to solute-solvent and solvent-solvent interactions.
\end{abstract}

Keywords: sodium sulfacetamide; solution calorimetry; van’t Hoff method.

\section{INTRODUCCIÓN}

La sulfacetamida sódica (SCM-Na, Figura 1) es un antibiótico bacteriostático de amplio espectro perteneciente a la familia de las sulfonamidas. Su actividad farmacológica se basa en la analogía estructural con el ácido $p$-amino benzoico (PABA), involucrado en la síntesis de ácido dihidrofólico. Este agente quimioterápico se encuentra disponible en formulaciones de administración oftálmica $(10 \% \mathrm{p} / \mathrm{v})$ y es ampliamente utilizado en el tratamiento de diversas infecciones de la conjuntiva y de la córnea, causadas por diversas bacterias; entre ellas, Staphylococcus aureus, Streptococcus pneumoniae, Streptococcus viridans, Escherichia coli, Haemophilus influenzae, Klebsiella/Enterobacter y Pseudomona aeruginosa. ${ }^{1}$<smiles>CC(=O)N([18O])O[Na]</smiles>

Figura 1. Estructura molecular de la sulfacetamida sódica

El conocimiento de las diferentes propiedades fisicoquímicas de fármacos en solución acuosa es fundamental en los procesos de preformulación y formulación de nuevas formas farmacéuticas. ${ }^{2}$ En particular, las propiedades termodinámicas de disolución de los diferentes componentes presentes en las formulaciones líquidas adquieren gran relevancia puesto que permiten estimar la estabilidad física de las mismas. ${ }^{3}$

\footnotetext{
*e-mail: fmartinezr@unal.edu.co
}

En general, el calor de solución de los fármacos $\left(\Delta H_{\text {soln }}^{0}\right)$ puede ser determinado por dos metodologías, una directa mediante la calorimetría y otra indirecta, estudiando las respectivas constantes de equilibrio $(K)$ en función de la temperatura, en este caso la solubilidad. Esta última estrategia es conocida como método de van't Hoff, y es de amplia utilización en la fisicoquímica farmacéutica. En dicho método, la pendiente de la curva de $\ln K$ vs. $1 / T$, multiplicada por $-R$ $\left(8,314 \mathrm{~J} \mathrm{~mol}^{-1} \mathrm{~K}^{-1}\right)$, se utiliza para determinar $\Delta H_{\text {soln }}^{0}$, si el soluto es del tipo no-electrolito. ${ }^{4} \mathrm{Si}$ bien los dos métodos pueden ser utilizados para determinar el cambio entálpico asociado a diferentes procesos fisicoquímicos, desde hace varios años la equivalencia entre ambos métodos (en particular, la calorimetría de titulación isotérmica) ha sido puesta en duda en el caso de algunas reacciones de interacción no covalente, tales como la autoasociación de proteínas para formar estructuras supramoleculares, ${ }^{5}$ la unión del 2'-monofosfato de citidina a la ribonucleasa $\mathrm{A},{ }^{6} \mathrm{y}$ la complejación del ion $\mathrm{Ba}^{2+}$ con el éter cíclico 18 -crown-6; $;^{7}$ si bien por el contrario, algunos estudios recientes corroboran su adecuada concordancia, en particular, para las dos últimas reacciones. ${ }^{8}$

La SCM-Na es un fármaco extremadamente soluble en agua y un número limitado de estudios termodinámicos investigando el proceso de disolución de acuerdo al método de van't Hoff han sido previamente reportados. ${ }^{9}{ }^{10}$ La caracterización de las interacciones existentes en solución por fármacos de tipo salino adquiere gran relevancia en términos de la estabilidad física y química de las formulaciones que contienen estos agentes, debido a que permitirían la identificación de posibles incompatibilidades entre componentes. ${ }^{10}$

Nuestros grupos de investigación dedican amplios esfuerzos al estudio sistemático de dichos fenómenos. Con el objeto de evaluar 
la equivalencia de la determinación llevada a cabo mediante ambas metodologías, en la presente comunicación, la $\Delta H_{\text {soln }}^{0}$ de SCM-Na en agua fue determinada experimentalmente utilizando calorimetría isoperibólica de disolución. ${ }^{11}$ Los valores obtenidos fueron comparados con los calculados mediante el método de van't Hoff a partir de los valores de solubilidad del fármaco en función de la temperatura. ${ }^{10}$

Los resultados demostraron que los valores calorimétricos de entalpía de disolución $\left(\Delta H_{\mathrm{soln}}^{\mathrm{cal}}\right)$ disminuyeron con el aumento de la concentración del fármaco y además, los valores obtenidos fueron significativamente diferentes de los reportados en los trabajos previos, los cuales fueron calculados mediante el método de van't Hoff $\left(\Delta H_{\mathrm{soln}}^{0-\mathrm{vH}}\right)$, excepto cuando se realiza la extrapolación a concentración $0 .{ }^{9,10}$ Así, se demostró que en el caso específico de las sales sódicas que son altamente solubles, el método de van't Hoff no sería un método adecuado para la determinación de $\Delta H_{\text {soln }}^{0}$. Este resultado podría explicarse en términos de las fuertes interacciones solutosoluto, presentes a las elevadas concentraciones requeridas por dicho método indirecto, además de las correspondientes interacciones del tipo soluto-solvente y solvente-solvente.

\section{PARTE EXPERIMENTAL}

\section{Reactivos}

Sulfacetamida sódica de Sigma (Saint Louis, E.U.), cloruro de potasio de Merck (Alemania), trihidroximetilamino metano (THAM) de Merck (Alemania) fueron de grado analítico y se almacenaron en desecador con $\mathrm{CaCl}_{2}$ como atmósfera desecante. El agua fue doblemente destilada obteniendo conductividad $<2 \mu \mathrm{S} \mathrm{cm}^{-1}$.

\section{Determinación calorimétrica de la entalpía de solución $\left(\Delta H_{\mathrm{soln}}^{\text {cal }}\right)$}

\section{Preparación de muestras}

Las cantidades de solvente (agua, $\mathrm{HCl}$ ) cercanas a 50,00 g y de soluto ( $\mathrm{KCl}$, THAM, SCM-Na) variando entre 0,0400 y $0,2500 \mathrm{~g}$ fueron determinadas de acuerdo a las dimensiones del calorímetro y las concentraciones se expresaron en términos de la relación: masa de soluto/masa de solvente. Se estudiaron cinco diferentes concentraciones a $25,0{ }^{\circ} \mathrm{C}$.

\section{Procedimiento calorimétrico}

Para las determinaciones calorimétricas se utilizó un calorímetro isoperibólico de solución, diseñado y construido en el Departamento de Química de la Universidad de los Andes, el cual ha sido presentado previamente en la literatura. ${ }^{11}$ Una vez controlada la temperatura del termostato, el procedimiento seguido consistió en lo siguiente: pesar la cantidad de soluto en la celda; pesar la cantidad de solvente en el vaso Dewar; colocar la celda tapada en el mecanismo de liberación; colocar el vaso Dewar de tal forma que el solvente cubra la celda, el termistor y la resistencia de calibración; colocar el calorímetro dentro del termostato y encender el sistema de agitación; incubar separadamente el solvente y el soluto durante $1 \mathrm{~h}$ para permitir la termostatización. En este período de tiempo se realiza la captura de datos para verificar el equilibrio térmico del solvente puro; accionar el sistema disparador de la celda interna para liberar el soluto en el solvente, registrando el cambio térmico que ocurre tras la reacción. Esperar hasta la estabilización de los datos y realizar un calentamiento similar en magnitud al cambio de voltaje ocurrido durante el proceso de solución; realizar calentamientos adicionales utilizando la misma metodología del paso anterior. Con estas calibraciones repetidas, posteriormente calcular el valor del $\Delta H_{\text {soln }}^{\mathrm{cal}}$ correspondiente al proceso de solución.

\section{Tratamiento de los datos}

La extrapolación de los datos calorimétricos a concentración 0 mol kg-1 de SCM-Na $\left(\Delta H_{\text {soln }}^{0 \text {-cal }}\right)$ fue realizada mediante regresión parabólica por el método de los mínimos cuadrados, utilizando los valores promedios y las respectivas incertidumbres experimentales.

\section{RESULTADOS Y DISCUSIÓN}

La calorimetría de solución se ha empleado en el campo farmacéutico para estudiar diferentes fenómenos relacionados con la preformulación y la formulación de medicamentos, por lo cual su utilidad práctica en el diseño de productos farmacéuticos tiene una relevancia previamente demostrada. ${ }^{12}$

Para demostrar la confiabilidad del calorímetro, se realizaron determinaciones de diferentes calores de reacción, empleando como sistemas de referencia al $\mathrm{KCl}+\mathrm{H}_{2} \mathrm{O}$ y al THAM $1,0 \mathrm{~N}+\mathrm{HCl}$, practicando cinco calentamientos en cada determinación. Los resultados obtenidos experimentalmente se presentan en la Tabla 1 y fueron comparados contra los datos reportados en literatura, ${ }^{12-15}$ encontrándose buena concordancia.

En cuanto a las determinaciones calorimétricas realizadas con SCM-Na se observó que los valores de entalpía de solución son positivos a todas las concentraciones evaluadas, además de que presentan un comportamiento no lineal en función de la molalidad, con pendiente negativa, indicando que esta propiedad termodinámica disminuye con el aumento en la concentración (Tabla 2, Figura 2). Mediante extrapolación a concentración cero de la respectiva ecuación parabólica ajustada por el método de los mínimos cuadrados se determinó la entalpía molar de solución a dilución infinita $\left(\Delta H_{\text {soln }}^{0 \text {-cal }}\right)$, siendo esta de 17,8 $\pm 0,2 \mathrm{~kJ} \mathrm{~mol}^{-1}$ (Tabla 2).

De otro lado, como se indicó previamente la solubilidad de la SCM-Na en medio acuoso y en función de la temperatura ha sido estudiada por Labastidas y Martínez, quienes reportaron valores considerablemente altos para esta propiedad $\left(3,231 \mathrm{~mol} \mathrm{~kg}^{-1}\right.$ a $\left.25,0^{\circ} \mathrm{C}\right) .{ }^{10}$ Una estrategia muy usada en el estudio fisicoquímico de compuestos

Tabla 1. Verificación del calorímetro

\begin{tabular}{|c|c|c|c|}
\hline \multirow{2}{*}{ Sistema } & \multirow{2}{*}{$\Delta H_{\text {reacción }}^{0 \text {-cal }}$ obtenido $\left(\mathrm{kJ} \mathrm{mol}^{-1}\right)$} & \multicolumn{2}{|c|}{$\Delta H_{\text {reacción }}^{0 \text {-cal }}$ literatura } \\
\hline & & Valor $\left(\mathrm{kJ} \mathrm{mol}^{-1}\right)$ & Ref. \\
\hline \multirow{3}{*}{$\mathrm{KCl}+\mathrm{H}_{2} \mathrm{O}^{\mathrm{a}}$} & \multirow{3}{*}{$17,31(0,03)^{a}$} & $17,58(0,05)$ & 13 \\
\hline & & $17,584(0,017)$ & 14 \\
\hline & & $17,32(0,02)$ & 12 \\
\hline \multirow{2}{*}{$\mathrm{THAM}+\mathrm{HCl}$} & \multirow{2}{*}{$-29,76(0,05)$} & $-29,75(0,02)$ & 15 \\
\hline & & $-29,72(0,02)$ & 12 \\
\hline
\end{tabular}

${ }^{\mathrm{a}}$ En el sistema $\mathrm{KCl}+\mathrm{H}_{2} \mathrm{O}, \Delta H_{\text {reacción }}^{0 \text {-cal }}$ corresponde a $\Delta H_{\text {soln }}^{0}$ 
Tabla 2. Calor de disolución de la SCM-Na en función de la concentración molal

\begin{tabular}{lc}
\hline SCM-Na $\left(10^{3} \mathrm{~mol} \mathrm{~kg}^{-1}\right)$ & $\Delta H_{\text {soln }}^{\text {cal }}\left(\mathrm{kJ} \mathrm{mol}^{-1}\right)$ \\
\hline $0,00^{\mathrm{a}}$ & $17,8(0,2)^{\mathrm{a}}$ \\
3,29 & $15,71(0,06)$ \\
6,63 & $13,58(0,10)$ \\
9,70 & $12,54(0,05)$ \\
13,03 & $11,62(0,09)$ \\
16,97 & $10,76(0,03)$ \\
\hline
\end{tabular}

${ }^{\mathrm{a}} \Delta H_{\text {soln }}^{\text {cal }}=\Delta H_{\text {soln }}^{0}$ Obtenido por extrapolación a concentración $0 \mathrm{~mol}$ $\mathrm{kg}^{-1}$ en la ecuación parabólica.

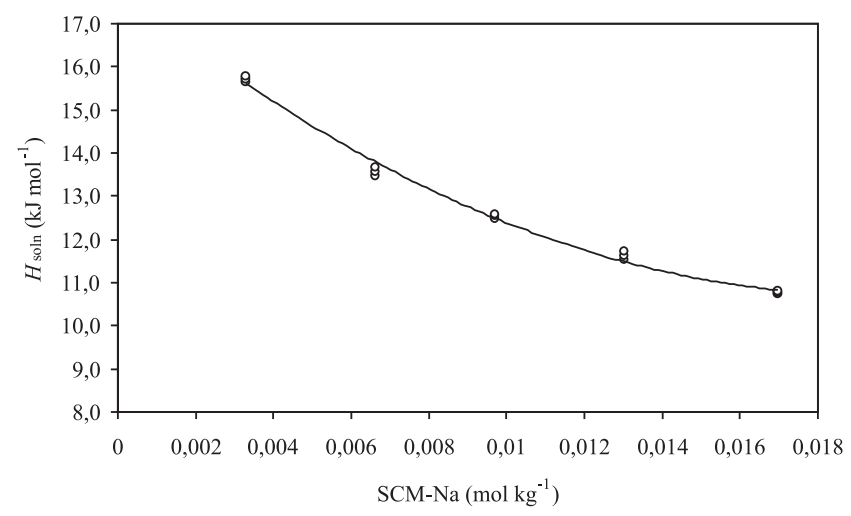

Figura 2. Entalpía de solución calorimétrica de la SCM-Na en función de la concentración molal. La ecuación parabólica es: $\Delta H_{\text {soln }}=17,81(0,19)$ $-731(42) \times m+18800(2026) \times m^{2}$, con $r^{2}$ ajustado: 0,9934 y error típico: 0,1562

de interés farmacéutico, en particular de fármacos, es el análisis de la variación de la solubilidad en función de la temperatura, mediante el método de van't Hoff. Como se indicó previamente, este método permite obtener el cambio entálpico estándar aparente de solución $\left(\Delta H_{\mathrm{soln}}^{\mathrm{omr}}\right)$. En el caso del fármaco estudiado (considerado como un electrolito del tipo uni-univalente), asumiendo completa disociación e ignorando las posibles interacciones interiónicas, la Ecuación de van't Hoff toma la forma:

$$
\left(\frac{\partial \ln m}{\partial T^{-1}}\right)_{P}=-\frac{\Delta H_{\mathrm{soln}}^{0 m-\mathrm{vH}}}{2 R}
$$

En la cual, los valores de solubilidad se expresan en la escala de molalidad para este caso particular. La Figura 3 corresponde al gráfico de van't Hoff, obtenido al procesar los datos presentados por Labastidas y Martínez. ${ }^{10} \mathrm{El}$ valor de $\Delta H_{\text {soln }}^{\mathrm{omvH}}$ se calculó entonces, como: $-2 R \times b$ (donde $b$ es la pendiente obtenida), obteniéndose un valor de $15,8 \pm 0,4 \mathrm{~kJ} \mathrm{~mol}^{-1}$ por este método.

Los resultados calorimétricos obtenidos (Tabla 2) corroboran lo presentado previamente en la literatura, en cuanto a que el proceso de solución de este fármaco es de naturaleza endotérmica. ${ }^{10}$ Además, al comparar los valores de $\Delta H_{\text {soln }}^{0}$ hallados por el método de van't Hoff en escala de molalidad $\left(15,8 \pm 0,4 \mathrm{~kJ}^{\mathrm{mol}}{ }^{-1}\right)$ y a dilución infinita por el método calorimétrico $\left(17,8 \pm 0,2 \mathrm{~kJ} \mathrm{~mol}^{-1}\right)$, se puede observar que no se presenta una amplia divergencia entre los dos resultados; lo cual aparentemente indicaría que el primer método sería válido, dentro de la incertidumbre experimental, para este tipo de determinaciones, en el estudio del proceso de disolución de las sales sódicas de estos fármacos. Sin embargo, es necesario tener en cuenta que en el estudio termodinámico de

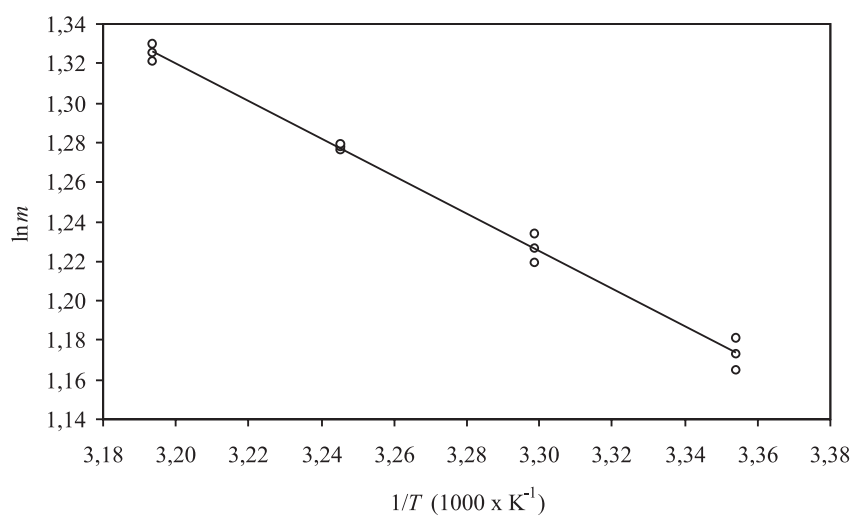

Figura 3. Gráfico de van't Hoff de la solubilidad de la SCM-Na expresada en molalidad. La regresión lineal obtenida es: $\ln m=4,36(0,08)-951$ (26) /T, con $r^{2}$ ajustado: 0,9920 y error típico: 0,0053

soluciones, a través de datos de solubilidad, además de considerar las interacciones soluto-solvente, también se deben considerar las respectivas interacciones soluto-soluto. Así, en el caso presente adquieren gran relevancia debido a los altos valores de solubilidad, que en la escala de fracción molar varían desde 0,0550 hasta 0,$0635 ; ;^{10}$ los cuales son muy superiores a los considerados como de alta dilución $\left(X_{2}<0,0001\right)^{16}$. De otro lado, las determinaciones calorimétricas en el presente trabajo, fueron realizadas en un rango de concentración moderadamente diluido, con lo cual se pretendió minimizar las interacciones soluto-soluto, además de que se hizo la extrapolación a dilución infinita con lo que predominan las interacciones soluto-solvente y solvente-solvente. Por todo lo anteriormente expuesto, es necesario tener precaución en el momento de la interpretación de las cantidades termodinámicas obtenidas por los dos métodos, que si bien son casi concordantes, es claro que los procesos globales de disolución involucrados en los dos casos se diferencian en el hecho de que el método de van't Hoff está incluyendo adicionalmente a las correspondientes interacciones soluto-soluto, las cuales conducen a la formación de estructuras inter-iónicas grandes del tipo racimo. ${ }^{17}$

Así mismo, debe considerarse que el método de van’t Hoff presenta limitaciones para hallar los valores de $\Delta H_{\text {soln }}^{0 \text {-vH }}$, en el caso de soluciones concentradas, puesto que estos valores deberían ser muy similares entre sí, independientemente de la escala de concentración utilizada en los cálculos, sin embargo como se demostró previamente en la literatura, en el caso de la SCM-Na los valores reportados de $\Delta H_{\text {soln }}^{0 \text {-vH }}$, son $9,8 \pm 0,3 \mathrm{~kJ} \mathrm{~mol}^{-1}$ y $14,9 \pm 0,4 \mathrm{~kJ} \mathrm{~mol}^{-1}$, para las escalas de molaridad y fracción molar, respectivamente, ${ }^{10}$ los cuales, de hecho no son comparables. Estos últimos resultados aparentemente contradictorios podrían explicarse en términos del volumen ocupado por el soluto en la solución saturada; ya que como puede observarse, los valores entálpicos obtenidos en las escalas de molalidad y de fracción molar son muy similares entre sí, mientras que si éstos dos se comparan con el obtenido en la escala de molaridad, no hay concordancia alguna, como se expresó ya anteriormente. Para ilustrar este efecto volumétrico, en la Tabla 3 se presentan los volúmenes molares aparentes de la SCM-Na y las respectivas fracciones volumétricas ocupadas por el fármaco y el agua en la concentración de saturación a diferentes temperaturas. Estos valores se calcularon a partir de los datos presentados previamente en la literatura para las soluciones saturadas. ${ }^{10}$

En particular, la densidad de las soluciones saturadas $\left(\rho_{\text {soln }}\right)$ se calculó a partir de los valores de solubilidad en fracción molar $\left(X_{2}\right)$ y en molaridad $(C)$, mediante la Ecuación $2:^{10}$ 
Tabla 3. Solubilidad porcentual másica de la SCM-Na $\left(\%\right.$ p/p), densidad de la solución saturada $\left(\rho_{\text {soln }}\right)$ y del agua $\left(\rho_{1}\right)$, volumen molar aparente de la SCM-Na a saturación $\left(\phi_{V}^{\text {sat }}\right)$ y fracciones volumétricas de la SCM-Na $\left(f_{\text {SCM-Na }}\right)$ y del agua $\left(f_{1}\right)$ a saturación a diferentes temperaturas

\begin{tabular}{lcccccc}
\hline Temp. $\left({ }^{\circ} \mathrm{C}\right)$ & $\mathrm{SCM}-\mathrm{Na}(\% \mathrm{p} / \mathrm{p})^{\mathrm{a}}$ & $\rho_{\text {soln }}\left(\mathrm{g} \mathrm{cm}^{-3}\right)$ & $\rho_{1}\left(\mathrm{~g} \mathrm{~cm}^{-3}\right)^{\mathrm{b}}$ & $\phi_{V}^{\text {sat }}\left(\mathrm{cm}^{3} \mathrm{~mol}^{-1}\right)$ & $f_{\text {SCM-Na }}$ & $f_{1}$ \\
\hline 25,0 & 43,28 & 1,2002 & 0,9970 & 144,3 & 0,317 & 0,683 \\
30,0 & 44,62 & 1,2045 & 0,9957 & 145,1 & 0,330 & 0,670 \\
35,0 & 45,88 & 1,2100 & 0,9940 & 145,2 & 0,341 & 0,659 \\
40,0 & 47,06 & 1,2127 & 0,9922 & 146,1 & 0,353 & 0,647 \\
\hline
\end{tabular}

${ }^{a}$ ref.10; bref. 18

$\rho_{\text {soln }}=\frac{C\left[M_{1}\left(1-X_{2}\right)+M_{2} X_{2}\right]}{1000 X_{2}}$

En la cual, $M_{1}$ y $M_{2}$ son las masas molares del agua y de la SCM$\mathrm{Na}$, siendo estas, 18,02 y $236,23 \mathrm{~g} \mathrm{~mol}^{-1}$, respectivamente. ${ }^{10}$ Mientras que el volumen molar aparente de la SCM-Na a saturación $\left(\phi_{V}^{\text {sat }}\right)$, se calculó mediante la Ecuación 3:19

$\phi_{V}^{\text {sat }}=\frac{M_{2}}{\rho_{\text {soln }}}+\frac{1000\left(\rho_{1}-\rho_{\text {soln }}\right)}{\rho_{1} \rho_{\text {soln }} m}$

En la cual, $\rho_{1}$ es la densidad del agua y $m$ es la solubilidad del fármaco expresada en la escala de molalidad. Finalmente, utilizando la composición porcentual y los volúmenes específicos aparentes en saturación, calculados como el cociente: $\phi_{V}^{\text {sat }} / M_{2}$, se calcularon las respectivas fracciones volumétricas de soluto y solvente en las soluciones saturadas. Todos estos valores se presentan en la Tabla 3.

Se observa que la fracción volumétrica del soluto es superior a 0,30 y además se incrementa con la temperatura, siendo proporcional al incremento en la solubilidad, por lo que consecuentemente la fracción de agua se ve disminuida. En este punto debe resaltarse que en el cálculo de las fracciones volumétricas sería más riguroso utilizar los valores de volumen molar parcial a saturación $\left(V_{2}^{\text {sat }}\right)$ en lugar de los valores $\phi_{V}^{\text {sat }}$, pero esto a su vez requeriría el estudio de $\phi_{V}$ en función de la concentración a las diferentes temperaturas, ${ }^{19}$ datos que por el momento no están disponibles. Sin embargo, en una primera aproximación, el uso de $\phi_{V}^{\text {sat }}$ es aceptable para estos fines prácticos.

Además, calculando el cociente entre los valores entálpicos de van't Hoff para las escalas de molaridad y fracción molar $\left(9,8 \mathrm{~kJ} \mathrm{~mol}^{-1}\right.$ / 14,9 $\mathrm{kJ} \mathrm{mol}^{-1}$ ), se obtiene el valor 0,658 , el cual es coincidente con la fracción volumétrica del agua a $35,0{ }^{\circ} \mathrm{C}$ (Tabla 3); lo cual resulta muy interesante, y si bien la explicación de este resultado no es muy clara, podría presumirse un efecto volumétrico sobre los valores de $\Delta H_{\mathrm{soln}}^{\mathrm{o}-\mathrm{vH}}$ debido a la disminución de la proporción de agua en las soluciones concentradas.

En un trabajo más completo, se podría determinar calorimétricamente la entalpía de disolución a diferentes temperaturas, así como la solubilidad del fármaco a otras temperaturas para ampliar el respectivo intervalo, por ejemplo entre 5,0 y $45,0^{\circ} \mathrm{C}$ (intervalo tradicionalmente estudiado mediante calorimetría de titulación termométrica, según la literatura), ${ }^{5-8}$ para verificar si en el proceso de disolución acuosa se presenta algún cambio en la capacidad calorífica $\left(\Delta C_{p}\right)$, como ha sido descrito para otros fenómenos fisicoquímicos, ${ }^{5-8}$ y que podría evidenciar posibles cambios en el mecanismo involucrado en el proceso. Así mismo, se debería evaluar la Ecuación 1 utilizando los valores de actividad termodinámica de la sal en condiciones de saturación, para lo cual se requeriría conocer la variación de los coeficientes de actividad del soluto con la concentración, ya que a valores superiores de $1,0 \mathrm{~mol} \mathrm{~kg}^{-1}$ las expresiones usadas para calcular estos valores, tales como la ecuación ampliada de Debye-Hückel, ${ }^{20}$ no resultan válidas, requiriéndose de expresiones mucho más complejas, que a su vez requieren de valores empíricos que deben ser determinados experimentalmente. ${ }^{21}$

Actualmente en nuestros grupos de investigación se adelanta el estudio de los coeficientes osmóticos $(\phi)$ de diferentes sales orgánicas en medios acuosos, utilizando equipos isopiésticos, análogos al que fue desarrollado en el Laboratorio de Investigaciones Básicas de la Universidad Nacional de Colombia, y que ha sido descrito previamente en la literatura. ${ }^{22}$ Con los valores de $\phi$ se podrían calcular los coeficientes de actividad de los solutos $\left(\gamma_{2}\right)$, y con estos valores a su vez, se podría hallar la actividad termodinámica de la sal en las soluciones saturadas.

\section{CONCLUSIONES}

De todo lo anteriormente expuesto se puede concluir que el calor de disolución de la SCM-Na en agua a $25,0{ }^{\circ} \mathrm{C}$ es dependiente de la concentración molal y que si bien, los valores obtenidos calorimétricamente a dilución infinita y por el método de van't Hoff son bastante concordantes, es necesario tener precaución a la hora de considerarlos como equivalentes, ya que las interacciones intermoleculares e interiónicas presentes son diferentes en las dos clases de experimentos, debido principalmente a la alta solubilidad acuosa de este fármaco y con cuyos valores se procedió en el método de van't Hoff, mientras que en la calorimetría se utilizaron concentraciones relativamente bajas.

\section{AGRADECIMIENTOS}

Los autores agradecen a la DIB-DINAIN de la Universidad Nacional de Colombia y al Fondo de Investigación de Profesores Asistentes de la Universidad de Los Andes por la ayuda financiera y el préstamo de los equipos e instalaciones.

\section{REFERENCIAS}

1. Sridhar, M. S.; Gopinathan, U.; Garg, P.; Sharma, S.; Rao, G. N.; Surv. Ophthalmol. 2001, 45, 361; Nagaraja, P.; Sunitha, K. R.; Vasantha R. A.; Yathirajan H. S.; Eur. J. Pharm. Biopharm. 2002, 53, 187.

2. Pérez, D. C.; Guevara, C. C.; Cárdenas, C. A.; Pinzón, J. A.; Barbosa H. J.; Martínez, F.; Rev. Colomb. Cienc. Quím. Farm. 2003, 32, 116.

3. Pacheco, D. P.; Manrique, Y. J.; Martínez, F.; Fluid Phase Equilibr. 2007, 262, 23.

4. Mora, C. P.; Martínez, F.; J. Chem. Eng. Data 2007, 52, 1933.

5. Sutherland, J. W. H.; Proc. Natl. Acad. Sci. USA 1977, 74, 2002.

6. Naghibi, H.; Tamura, A.; Sturtevant, J. M.; Proc. Natl. Acad. Sci. USA 1995, 92, 5597.

7. Mizoue, L.S.; Tellinghuisen, J.; Biophys. Chem. 2004, 110, 15.

8. Horn, J. R.; Russell, D.; Lewis, E. A.; Murphy K. P.; Biochemistry 2001, 40, 1774.

9. Torres, D. R.; Vargas, E. F.; Martínez, F.; Resúmenes del XVII Congreso Farmacéutico Argentino, Villa Carlos Paz, Argentina, 2006. 
10. Labastidas, I.; Martínez, F.; Acta Farm. Bonaerense 2006, 25, 55.

11. Vargas, E. F.; Moreno, J. C.; Forero, J.; Parra, D. F.; J. Therm. Anal. Calorimetry 2008, 91, 659.

12. Ramos, R.; Gaisford, S.; Buckton, G.; Royall, P. G.; Yff, B. T. S.; O’Neill; M. A. A.; Int. J. Pharm. 2005, 299, 73; Conti, S.; Gaisford, S. Buckton, G.; Conte, U.; Thermochim. Acta 2006, 450, 56; Gaisford, S.; O’Neill, M. A. A.; Thompson, L.; Chan, K-L.; Hosp. Pharm. 2006, 13, 295; Conti, S.; Gaisford, S.; Buckton, G.; Maggi, L.; Conte, U.; Eur. J. Pharm. Biopharm. 2008, 68, 795.

13. Uriano, G. A.; Standard Reference Material 1655, Potassium Chloride, $\mathrm{KCl}$ ( cr) for Solution Calorimetry, National Bureau of Standards Certificate, 1981.

14. Wadso, I.; Goldberg, R.; Pure Appl. Chem. 2001, 73, 1625.

15. Hill, J. O.; Ojlund, G.; Wadso, I.; J. Chem. Thermodyn. 1969, 1, 111.

16. Klotz, I. M.; Rosenberg, R. M.; Chemical Thermodynamics: Basic Theory and Methods, $6^{\text {th }}$ ed., John Wiley \& Sons, Inc.: New York, 2000, p. 438-448; Bevan, J.; Boerio-Goates, J.; Chemical Thermodynamics: Principles and Applications, Academic Press: New York, 2000, p. 295 301.

17. Abascal, J. L. F.; Bresme, F.; Turq, P.; Mol. Phys. 1994, 81, 143; Lu, G. W.; Li, C. X. ; Tian, R.; Wang, Z. H.; Wang, W. C.; Fluid Phase Equilibr. 2004, 218, 77 .

18. Lide, D. R.; CRC, Handbook of Chemistry and Physics, $84^{\text {th }}$ ed., CRC Press LLC: Boca Raton, 2003, p. 5-6.

19. Martínez, F.; Gómez, A.; Ávila, C. M.; Acta Farm. Bonaerense 2002, 21, 107

20. Martin A.; Bustamante, P.; Chun, A. C. H.; Physical Pharmacy: Physical Chemical Principles in the Pharmaceutical Sciences, $4^{\text {th }}$ ed.; Lea \& Febiger: Philadelphia, 1993, p. 135-137.

21. Stokes, R. H.; Robinson, R. A.; J. Am. Chem. Soc. 1948, 70, 1870; Eigen, M.; Wicke, E.; J. Phys. Chem. 1954, 58, 702.

22. Amado, E.; Blanco, L. H.; Fluid Phase Equilibr. 2004, 226, 261. 